\title{
The role of quasi-momentum in the resonant dynamics of the atom-optics kicked rotor
}

\author{
Sandro Wimberger $\uparrow \S$ and Mark Sadgrove $\ddagger$ \\ †CNR-INFM and Dipartimento di Fisica Enrico Fermi, Università di Pisa, Largo \\ Pontecorvo 3, 56127 Pisa, Italy \\ $\ddagger$ Department of Physics, University of Auckland, Private Bag 92019, Auckland, New \\ Zealand
}

\begin{abstract}
We examine the effect of the initial atomic momentum distribution on the dynamics of the atom-optical realisation of the quantum kicked rotor. The atoms are kicked by a pulsed optical lattice, the periodicity of which implies that quasi-momentum is conserved in the transport problem. We study and compare experimentally and theoretically two resonant limits of the kicked rotor: in the vicinity of the quantum resonances and in the semiclassical limit of vanishing kicking period. It is found that for the same experimental distribution of quasi-momenta, significant deviations from the kicked rotor model are induced close to quantum resonance, while close to the classical resonance (i.e. for small kicking period) the effect of the quasimomentum vanishes.
\end{abstract}

PACS numbers: 42.50.Vk,32.80.Qk,05.45.Mt,05.60.-k

\section{Introduction}

The past decade has brought fascinating advances in the preparation and control of single particles [1]. Atoms can now be cooled down to a level where the effect of a single photon recoil can be measured experimentally [2]. Single atom dynamics can thus be controlled with high precision by introducing an external field in the form of an optical potential [3, 4].

A particular example of such a system, the atom-optics kicked rotor, has shed light on interesting and paradigmatic quantum effects including dynamical localisation [3] and quantum resonance [5, 6, 7, 8. In all such experiments, control of the initial conditions in phase space is essential. In particular, the impact of different momentum classes on the dynamics near quantum resonance was explained recently [9, 10]. The atoms are kicked by a spatially periodic potential which is pulsed on at a certain frequency. As dictated by standard Bloch theory, the spatial periodicity implies that the quasi-momentum for the centre-of-mass motion of each atom is conserved during the evolution. Quasimomentum is an intrinsically quantum variable which arises due to the translational $\S$ Corresponding author's e-mail: saw@pks.mpg.de 
symmetry of the problem [11. Since experiments with cold atoms typically use a broad, continuous distribution of quasi-momenta, the experimental data represents a result averaged over this initial distribution [9, 10, 12, 13.

The averaging over different momentum classes leads to significant deviations from the standard $\delta$-kicked rotor model [14, 15] which typically does not consider the additional control parameter introduced by the quasi-momentum. Such deviations have been experimentally observed, in particular at quantum resonance 9 and have been explained theoretically by means of a new pseudo-classical model introduced in [16] and applied to the usual $\delta$-kicked rotor in [10, 17].

In this paper, we use the same theoretical formalism to expose the innate similarities and surprising differences between the limit in which the exact quantum resonant driving is approached and the limit of vanishing kicking period. The former limit can be described using the pseudo-classical model from [10, 17] (with an effective Planck constant defined by the detuning from exact resonance), whilst the latter limit is the usual classical limit of the kicked rotor (with the scaled kicking period as the effective Planck constant). Our theoretical analysis of the experimental data focuses on the role of the quasi-momentum, which proves to be quite different in the two "classical" limits studied here.

\section{The Atom-Optics Kicked Rotor}

We consider a system of Caesium atoms in an optical standing wave (with wave number $k_{L}$ ) which is $\delta$-pulsed with period $\tau$. For sufficiently large detuning from the atomic absorption line, the Hamiltonian for an atom is given by [18]

$$
H\left(t^{\prime}\right)=\frac{p^{2}}{2}+k \cos (z) \sum_{t=0}^{N} \delta\left(t^{\prime}-t \tau\right),
$$

where $p$ is the atomic momentum in units of $2 \hbar k_{L}$ (i.e., in units of two-photon recoils), $z$ is the atomic position in units of $2 k_{L}, t^{\prime}$ is time and $t$ is the kick number. The scaled kicking period $\tau$ is defined by the equation $\tau=8 E_{R} T / \hbar$, where $E_{R}=\hbar^{2} k_{L}^{2} / 2 M$ is the recoil energy (associated with the energy change of a Caesium atom of mass $M$ after emission of a photon of wavelength $\lambda_{L}=2 \pi / k_{L}=852 \mathrm{~nm}$ ). The kicking strength of the system is given by $k=V_{0} \tau / \hbar$ where $V_{0}$ is the maximum potential depth created by the optical standing wave [3, 18].

Experimentally, momentum kicks are delivered to the atoms by an optical lattice which is created by a $150 \mathrm{~mW}$ diode laser injection locked to a lower power feedback stabilised source at $852 \mathrm{~nm}$. Kicking laser powers of up to $30 \mathrm{~mW}$ were employed for detunings of $500 \mathrm{MHz}$ from the $6 S_{1 / 2}(F=4) \rightarrow 6 P_{3 / 2}\left(F^{\prime}=5\right)$ transition of Caesium. For the experimental results presented in this paper, the average energy of the atomic ensemble was measured after up to 20 kicks. To control the pulse timing, a custom built programmable pulse generator was employed to gate an acousto-optic modulator which controlled the amount of kicking light reaching the atomic sample. Timing of 
the experiment was controlled by a real-time, software based computer system with a latency on the order of $10 \mu \mathrm{s}$.

For the classical resonance experiments reported here, the kicking pulse width was $320 \mathrm{~ns}$, whilst for the quantum resonance results, a $480 \mathrm{~ns}$ pulse width was used. In the classical limit of vanishing kicking period, the $\delta$-kick approximation is violated in the experiment (although for the small kick numbers and kicking strengths used here, our results do not show deviations from the $\delta$-kick theory [19, 20, 21]). As a consequence, it is possible to probe the dynamics at exact quantum resonance, but not at the exact classical limit, since the pulse period $\tau$ should always exceed the pulse width to ensure a reliable approximation to $\delta$-pulses.

The experimental sequence ran as follows: Atoms were released from the magnetooptical trap [2] and then kicked by a series of light pulses. A free expansion time of 12 ms was then allowed followed by "freezing" of the atomic motion in optical molasses and subsequent CCD imaging of the resultant atomic cloud [8]. Mean energies are extracted from the raw data by calculating the second moment of the experimentally measured momentum distribution of the atoms' centre-of-mass motion.

By exploiting the spatial periodicity of the Hamiltonian (11), the atomic dynamics along the $z$ axis can be reduced to that of a rotor on a circle by Bloch's Theorem [10]. This introduces the additional parameter $\beta \in[0,1)$ which represents the atomic quasimomentum - a constant of the motion by Bloch's theorem. The fractional part of the physical momentum $p$ in the units given above corresponds to the quasi-momentum which is practically uniformly distributed in the fundamental Brillouin zone defined by the periodic kick potential [10]. The one-kick propagation operator for a given atom is 10

$$
\hat{\mathcal{U}}_{\beta}=e^{-\mathrm{i} k \cos (\hat{\theta})} e^{-\mathrm{i} \tau(\hat{\mathcal{N}}+\beta)^{2} / 2},
$$

where $\theta=x \bmod (2 \pi)$, and $\hat{\mathcal{N}}=-\mathrm{i} d / d \theta$ is the angular momentum operator with periodic boundary conditions.

\section{Unifying Classical Description of Quantum and Classical Resonance}

The quantum dynamics in the two semiclassical limits studied here is approximated by the following map [12, 17]:

$$
\begin{aligned}
& I_{t+1}=I_{t}+\tilde{k} \sin \left(\theta_{t+1}\right), \\
& \theta_{t+1}=\theta_{t} \pm I_{t}+\ell \pi+\tau \beta \bmod (2 \pi),
\end{aligned}
$$

where $\tau=2 \pi \ell+\epsilon$ and $\tilde{k}=k|\epsilon|$, and $\ell=0,1,2$ ( \pm is the sign of $\epsilon$, and for $\ell=0$ only + is allowed). The above map is similar to the well-studied Standard Map [22] augmented by the term $\tau \beta$ which accounts for the experimental quasi-momentum distribution. Changing variables to $J= \pm I+\ell \pi+\tau \beta, \vartheta=\theta+\pi(1-\operatorname{sign}(\epsilon)) / 2$ formally gives the true Standard Map

$$
\begin{aligned}
J_{t+1} & =J_{t}+\tilde{k} \sin \left(\vartheta_{t+1}\right), \\
\vartheta_{t+1} & =\vartheta_{t}+J_{t} .
\end{aligned}
$$



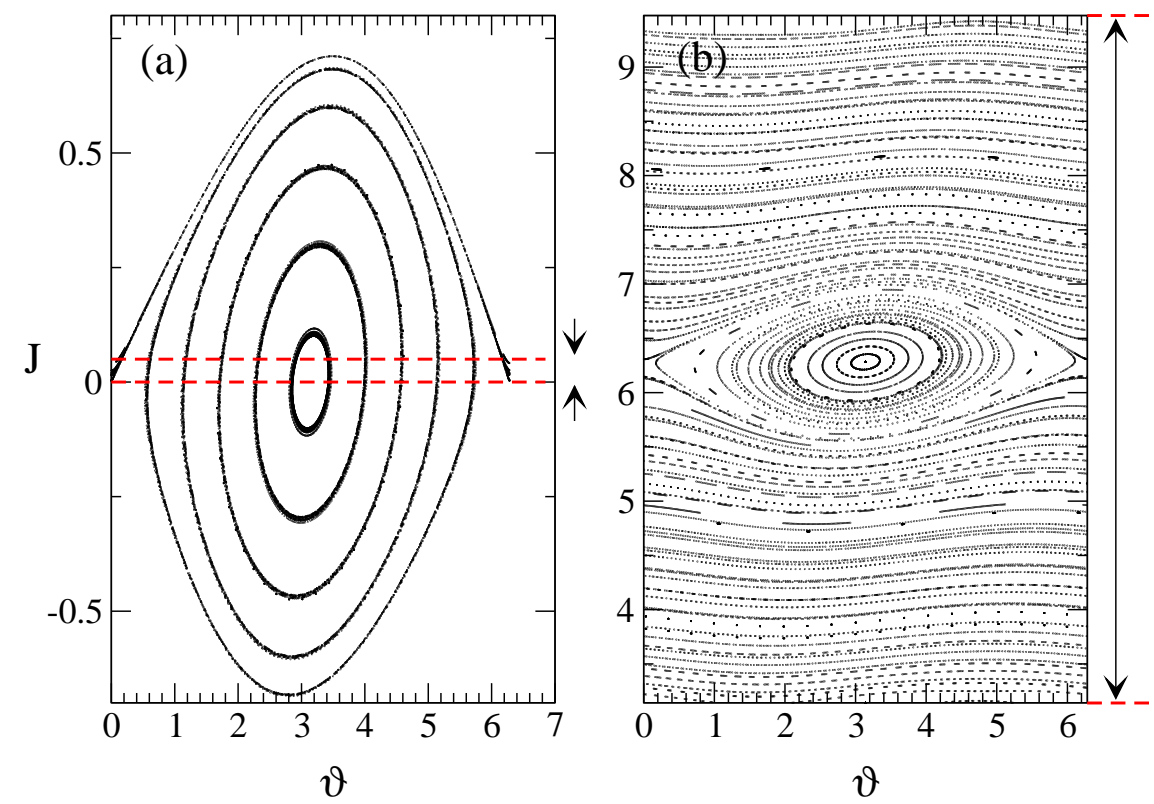

Figure 1. Phase space portrait generated by the map (4) for $k=2.5$ and $\epsilon=0.05$. The initial angles $\theta_{0}$ were uniformly distributed in $[0,2 \pi)$ whilst the initial momenta $J_{0}$ were taken from uniform distributions on the two different intervals $[0, \epsilon)$ (a) and $[\pi, 3 \pi+\epsilon)$ (b) as shown by the arrows in both figures. Note that the phase space is $2 \pi$-periodic along the $J$ axis.

The mean energy is calculated using the formula

$$
\left\langle E_{t, \epsilon}\right\rangle=\epsilon^{-2}\left\langle I_{t}^{2}\right\rangle / 2=\epsilon^{-2}\left\langle\delta J_{t}^{2}\right\rangle / 2, \quad \delta J_{t}=J_{t}-J_{0} .
$$

Although the map (44) is not explicitly dependent on the additional $\beta$ dependent term, we note that the initial conditions in momentum space are given by $J_{0}= \pm I_{0}+\pi \ell+\tau \beta$, i.e., they are defined by the initial choice of quasi-momentum $\beta$.

Two a priori quite different regimes are described by either of the two maps (31) or (44): firstly that for $\ell=0$, and $\tau \rightarrow 0$ and secondly that for $\ell>0, \tau \rightarrow 2 \pi \ell$, for integer $\ell$. In the case where $\ell=0$ we have $\epsilon=\tau$ and $J=\tau p$, with the physical momentum $p$ in units of two-photon recoils [12]. For integer $\ell>0$, the map in (44) approximates the dynamics near the fundamental quantum resonances occurring at $\tau=2 \pi \ell$. As shown in Refs. 10, 17, the one-kick propagator (2) may be rewritten in the form

$$
\hat{\mathcal{U}}_{\beta}(t)=e^{-\mathrm{i} \tilde{k} \cos (\hat{\theta}) /|\epsilon|} e^{-\mathrm{i} \hat{\mathcal{H}}_{\beta} /|\epsilon|},
$$

where $\epsilon=\tau-2 \pi \ell, \tilde{k}=|\epsilon| k, \hat{I}=|\epsilon| \hat{\mathcal{N}}$ and $\hat{H}_{\beta}=\frac{1}{2} \operatorname{sign}(\epsilon) \hat{I}^{2}+\hat{\mathrm{I}}(\pi \ell+\tau \beta)$. Considering $|\epsilon|$ to be an effective Planck constant, we see that the map given in Eq. (3) approximates the dynamics induced by (2) in both classical limits for $\epsilon \rightarrow 0$.

Figure 1 1emonstrates the essential difference between the two semiclassical limits studied here. In the case where $\ell=0$ (see Fig. 1 (a)), a uniform quasi-momentum distribution on $[0,1)$ leads to the initial momenta $J_{0}$ being uniformly distributed on the interval $\left[0, \sigma_{p} \epsilon\right)$, where $\sigma_{p}$ is the characteristic width of the initial atomic momentum 


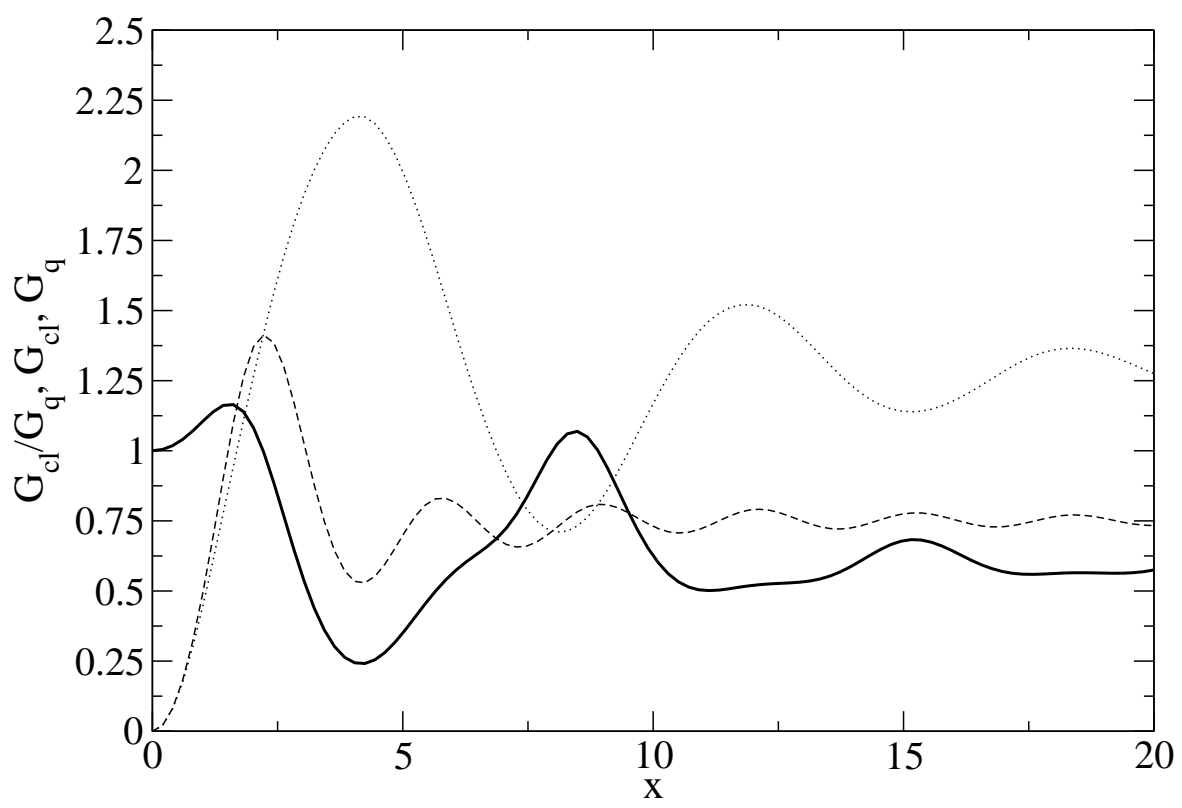

Figure 2. The ratio $G_{\mathrm{cl}} / G_{\mathrm{q}}$ (solid line) is shown along with the functions $G_{\mathrm{cl}}$ (dashed line) and $G_{\mathrm{q}}$ (dotted line) themselves. The ratio saturates to a constant for large $x$ after initial oscillations, as the classical and quantum resonance peaks decay at the same rate. The differences between the two scaling functions arise due to the different

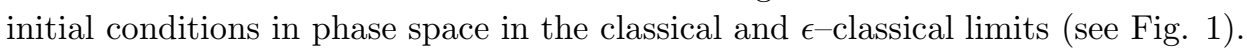

distribution in units of two-photon recoils. Therefore, for $\sigma_{p} \sim 1$, the initial momenta lie entirely within the region of phase space dominated by the nonlinear resonance island of the Standard Map . For $\ell=1$ (see Fig. 1(b)), and the same uniform quasi-momentum distribution, the initial momenta populate the full unit cell $[\pi, 3 \pi)$ in the periodic phase space which encompasses not only the nonlinear resonance island at $J=2 \pi$, but also regular "rotation" motion beyond it. Therefore the same experimental quasi-momentum distribution leads to different behaviour of the atomic ensemble in the two limits of $\ell=0$ and $\ell \neq 0$.

Based on the maps (31) and (41), useful results were previously derived for the analysis of experimental data [12, 17]. These results may be summarised by the following single parameter scaling functions which differ for the two limits of interest here. For $\ell=0$, the scaling function of the mean energy close to $\epsilon=\tau=0$ is given by

$$
\frac{\left\langle E_{t, \tau}\right\rangle}{\left\langle E_{t, 0}\right\rangle} \approx R_{c l}(x) \equiv \frac{2}{x^{2}} G_{\mathrm{cl}}(x),
$$

with $x=t \sqrt{k|\epsilon|}$ and the function $G_{\mathrm{cl}}$ defined by

$$
G_{c l}(x) \approx \frac{1}{2 \pi} \int_{0}^{2 \pi} \mathrm{d} \theta_{0} \bar{J}\left(x, \theta_{0}, J_{0}=0\right)^{2},
$$


where $\bar{J} \equiv J / \sqrt{\tilde{k}}$ is the momentum of the pendulum approximation to the dynamics generated by the map of Eq. (3) as defined previously in Ref. [10, 17].

For $\ell>0$, we have instead close to $\epsilon=0$

$$
\frac{\left\langle E_{t, \epsilon}\right\rangle}{\left\langle E_{t, 0}\right\rangle} \approx R_{q}(x) \equiv 1-\Phi_{0}(x)+\frac{4}{\pi x} G_{\mathrm{q}}(x),
$$

with different functions $\Phi_{0}$ and $G_{q}$. In this case, we have

$$
G_{q}(x) \approx \frac{1}{8 \pi} \int_{0}^{2 \pi} \mathrm{d} \theta_{0} \int_{-2}^{2} \mathrm{~d} J_{0} \bar{J}\left(x, \theta_{0}, J_{0}\right)^{2} .
$$

The difference between the two scaling functions $G_{\mathrm{cl}}$ and $G_{\mathrm{q}}$ may be seen in Fig. 2 where the ratio of the two functions is plotted along with the functions themselves. Although the functions have the same slope for small $x$, their forms differ in general and for large $x$, the ratio saturates to a constant less than 1 . The difference in the saturation values of the two $G$ functions arises from the different initial conditions in the the phase space of map 3 which apply in the classical and $\epsilon$-classical limits.

In the following section, we compare experimental data for the two different cases $\ell=0$ and $\ell=1,2$ guided by the theoretical results reviewed in the present section.

\section{Experimental vs. Theoretical Results}

In Figure 3, experimentally measured energies close to the classical and quantum resonances are plotted against the kick number. In both plots of this figure, the observed oscillatory behaviour may be understood in terms of the pendulum approximation to the dynamics of the map (41) as embodied by the functions $G_{\mathrm{cl}}(x)$ or $G_{\mathrm{q}}(x)$. [10, 12, 17, 23]. For small times $(t<5$ for the data in Fig. 3 (a)), the energy growth near the classical resonance is ballistic, i.e., the energy grows quadratically in time.

We note that ballistic motion is also predicted to occur at quantum resonance for an atomic ensemble with a very narrow initial momentum distribution [24, 25]. But the broad initial momentum distribution present in cold atom experiments as discussed here, typically leads to a uniform distribution of all possible values of quasi-momentum [9, 10]. In terms of the classical model reviewed in the previous section, these experimental initial conditions correspond to initial momenta distributed over the full phase space cell, as shown in Fig. 1 (b). The majority of the atoms obey rotational motion with almost constant energies (see Fig. 1(b)), whilst only a small sub-class follows the motion inside the nonlinear resonance island, which for a finite time (depending on the detuning $\epsilon$ ) supports ballistic energy growth [10, 17].

The connection between the dynamics in the classical limit and that for a quantum particle starting from a momentum eigenstate is found in the term $\tau \beta$ in the map (3). We see that this term may become zero in either of the following limits: $\tau \rightarrow 0$ or $\beta \rightarrow 0$. In both cases, the effect is to regain ballistic energy growth. The inset in Fig. 3 (a) shows a detailed scan of the mean energy near the classical resonance as $\tau \rightarrow 0$ which emphasises the rapid energy growth seen in this regime associated with the ballistic classical resonance. 


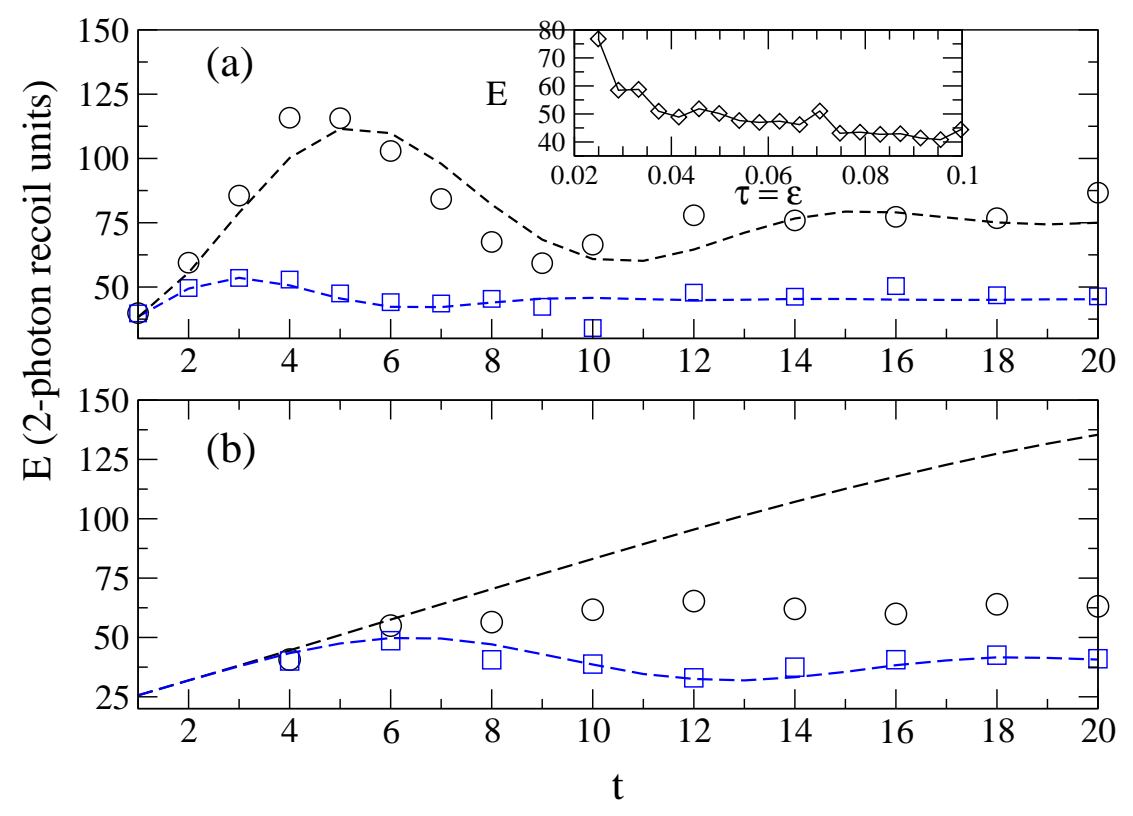

Figure 3. Experimental measurements of the mean energy as a function of kick number for $k \approx 5$, taken for small values of the detuning $\epsilon$ in the limits $\tau \rightarrow 0$ (a) and $\epsilon \rightarrow 0$ for $\ell=1$ (b). In particular, we have (a) $\epsilon=0.033$ (circles) and $\epsilon \approx 0.08$ (squares), and (b) $|\epsilon| \lesssim 0.005$ (circles) and $\epsilon \approx 0.08$ (squares), along with classical simulations using the map (44) (dashed lines). We note the oscillatory nature of the energy curve for finite detuning $\epsilon$, which may be viewed as a consequence of the dynamics represented by the phase spaces in Fig. 1 The inset in (a) shows a detailed experimental scan of the classical resonance peak as $\tau=\epsilon \rightarrow 0$, for $k \approx 2.5$ and after $t=5$ kicks.

Figure 3 (b) shows mean energy measurements at exact quantum resonance (circles) and for $\epsilon \approx 0.08$ along with $\epsilon$-classical simulation results (dashed lines). For the same experimental momentum distribution, only linear mean energy growth is predicted to occur at exact quantum resonance. Additionally, the data shown here demonstrate a practical problem which arises from the uniform distribution of quasi-momenta over the first Brillouin zone. Because only the quasi-momentum classes $\beta \approx 1 / 2$ (for $\ell=1$ ) and $\beta \approx 0,1 / 2$ (for $\ell=2$ ) experience quantum resonant dynamics [9, 10, 15], only a small number of resonant atoms are responsible for the linear growth of the ensemble mean energy. The measurement of the mean energy at exact quantum resonance is therefore experimentally very challenging since the signal-to-noise ratio is low for the small population of resonant atoms [5, 7, 9, 10]. This is the most likely cause of the apparent saturation of energy growth in the quantum resonance case as seen in Fig. [3 (b) where the experimental mean energy (circles) noticeably deviates from the expected linear growth (dashed line). Indeed, inspection of the experimental momentum distributions for the on-resonance data reveals that the characteristic ballistic wings associated with resonant atoms [9] are not resolved for kick numbers greater than about 6 in these experiments.

By comparison with the data in 3 (a) for the classical resonance, we see that, even though the maximum energy is much larger than that measured at quantum resonance 


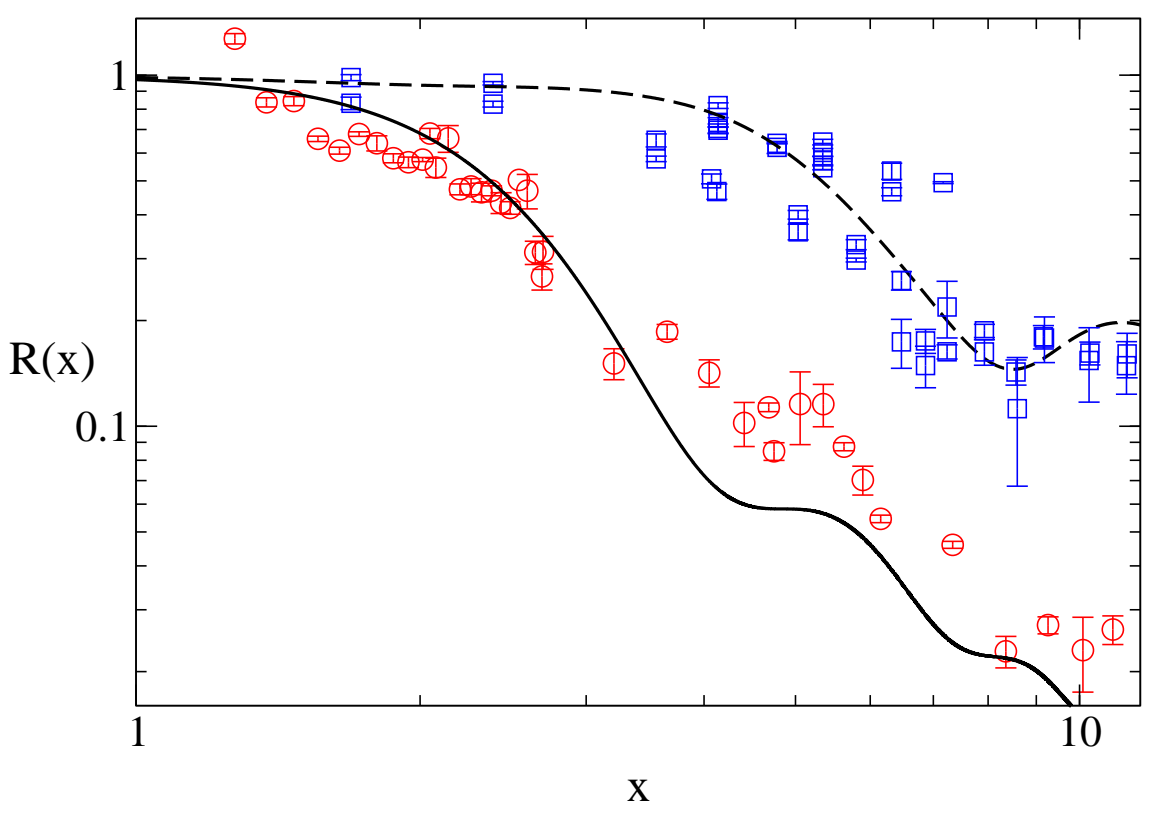

Figure 4. Rescaled experimental mean energies near classical resonance (circles), and the quantum resonances at $\tau=2 \pi$ and $4 \pi$ (squares). In particular, the circles for $x \lesssim 3$ are rescaled data from the inset of Fig. 3 (a). The mean energies have been scaled by the theoretical peak height of the resonances, i.e., by $k^{2} t^{2} / 4$ for the classical resonance [12] and $k^{2} t / 4$ for the quantum resonance data [10 17. The scaling functions for the classical (7) and quantum resonances (9) are shown as a solid line and a dashed line respectively. The narrower width of the classical resonance peak is immediately apparent. This figure also shows the utility of the scaling function in the comparison of data which is meaningful in the scaled units even for a wide range of the three parameters: here for $k \approx 2.5$ and $k \approx 5(\ell=0)$ and $k \approx 5(\ell=1,2), 0.033 \leq \epsilon \leq 0.1$ $(\ell=0)$ and $0.03 \leq \epsilon<0.3(\ell=1,2)$, and $3 \leq t \leq 16$. Error bars represent statistical fluctuations over three independent experiments.

for the same number of kicks, the initial quadratic mean energy growth can easily be resolved since practically the entire atomic ensemble experiences resonant energy growth in this regime. This is precisely because as $\tau$ tends to zero, the $\beta$ dependence of the map (3) is removed as the term $\tau \beta$ vanishes at the same rate as $\tau$.

Lastly, Fig. 4 shows rescaled data from experimental measurements for various experimental parameters with $\ell=0$ (circles) and $\ell=1,2$ (squares). The data taken in the classical case $(\ell=0)$ falls on or close to the classical scaling function (solid line in Fig. (3) and that, likewise, the data taken for $\ell=1,2$ falls on or near the quantum scaling curve (dash-dotted line). The narrower nature of the classical resonance peak is emphasised by this plot. The dense set of points (circles) shown for $x \lesssim 3$ in the classical case come from the data shown in the inset of in Fig. 3. (a). This data provides a detailed confirmation of the classical scaling function's validity for smaller values of $x$ than previously observed experimentally [23]. Somewhat surprisingly, it is found that the $\delta$-kicked rotor theory holds even in a regime of $x$ for which the spacing between kicking pulses is comparable to the width of the pulses themselves [12. The smallest value of the kicking period $\tau$ for which the $\delta$-kicked model remained valid in these experiments 
was $\tau=0.033$ which, for a kicking strength $k \approx 5$ and $t=5$, corresponds to $x \approx 2$. For larger $x$, the data points show more scatter because of systematic fluctuations in the initial momentum spread and the difficulty in observing the peak very close to resonance for a larger number of kicks [23].

\section{Conclusion}

We have demonstrated the effect of averaging over a uniform quasi-momentum distribution in two different semiclassical limits of the atom-optics kicked rotor. For the same experimental quasi-momentum distribution, the true classical limit gives rise to ballistic energy growth whereas in the pseudo-classical limit approximating quantum resonance only linear growth occurs.

This difference is explained by considering the inclusion of the quasi-momentum dependent term $\tau \beta$ in the theoretical description. If this term approaches zero, which may be accomplished either by performing the classical limit $\tau \rightarrow 0$ or starting with a very narrow momentum distribution such as that provided by a Bose-Einstein condensate [25], ballistic energy growth is recovered. However, for standard atom-optics kicked rotor experiments using cold atoms only linear energy growth is predicted at quantum resonance since the quasi-momentum $\beta$ is uniformly distributed in the entire Brillouin zone.

The classical theory of Section 3 of the near resonant dynamics thus unifies the description of quantum and classical resonance behaviour of the atom-optics kicked rotor, and is elegantly summarised by two classical one-parameter scaling laws for the classical and quantum resonance peaks. These laws are very useful for a detailed analysis of experimental results in regimes in which measurements are limited by the signal-tonoise ratio.

\section{Acknowledgments}

The authors would like to thank Rainer Leonhardt and Scott Parkins for helpful discussions. Additionally, we are grateful to Andreas Buchleitner and Javier Madroñero for their hospitality and logistical support at the Max Planck Institute for the Physics of Complex Systems in Dresden. M.S. was supported by The Tertiary Education Commission of New Zealand. S.W. thanks the organisers of the International Workshop on "Aspects of Quantum Chaotic Scattering" (Dresden, 2005) for providing a stimulating atmosphere and partial financial support, as well as the Alexander von Humboldt Foundation (Feodor-Lynen Program) for funding.

\section{References}

[1] See, e.g., Varcoe BTH, Brattke S, Weidinger M and Walther H, 2000 Nature 403 743; McKeever J, Boca A, Boozer AD, Buck JR and Kimble HJ 2003 Nature 425, 268; Maunz P, Puppe T, Schuster I, Syassen N, Pinkse PWH and Rempe G 2004 Nature 428 50; Riebe M, Häffner H, 
The role of quasi-momentum in the resonant dynamics of the AOKR

Roos CF, Hänsel W, Benhelm J, Lancaster GPT, Körber TW, Becher C, Schmidt-Kaler F, James DFV and Blatt R 2004 Nature 429 734; Barrett MD, Chiaverini J, Schätz T, Britton J, Itano WM, Jost JD, Knill E, Langer C, Leibfried D, Ozeri R and Wineland DJ 2004 Nature 429 737; Schrader D, Dotsenko I, Khudaverdyan M, Miroshnychenko Y, Rauschenbeutel A and Meschede D 2004 Phys. Rev. Lett. 93150501

[2] Monroe C, Swann W, Robinson H and Wieman C 1990 Phys. Rev. Lett. 651571

[3] Moore FL, Robinson JC, Bharucha CF, Sundaram B and Raizen MG 1995 Phys. Rev. Lett. 75 4598

[4] Anderson BP and Kasevich MA 1998 Science 282 1686; Steck DA, Oskay WH and Raizen MG 2001 Science 293 274; Hensinger WK, Häffner H, Browaeys A, Heckenberg NR, Helmerson K, C. Mckenzie C, Milburn GJ, Phillips WD, Rolston SL, Rubensztein-Dunlop H and Upcroft B 2001 Nature 412 52; Greiner M, Mandel O, Esslinger T, Hänsch TW and Bloch I 2002 Nature 41539

[5] Oskay WH, Steck DA, Milner V, Klappauf BG and Raizen MG 2000 Opt. Commun. 179137

[6] d'Arcy MB, Godun RM, Oberthaler MK, Cassettari D and Summy GS 2001 Phys. Rev. Lett. 87 074102

[7] d'Arcy MB, Godun RM, Oberthaler MK, Summy GS, Burnett K and Gardiner SA 2001 Phys. Rev. E 64056233

[8] Sadgrove M, Hilliard A, Mullins T, Parkins S and Leonhardt R 2004 Phys. Rev. E 70036217

[9] d'Arcy MB, Godun RM, Summy GS, Guarneri I, Wimberger S, Fishman S and Buchleitner A 2004 Phys. Rev. E 69027201

[10] Wimberger S, Guarneri I and Fishman S 2003 Nonlinearity 161381

[11] Ashcroft NW, Mermin ND, Solid State Physics 1976 (Holt, Rinehart and Winston, Philadelphia)

[12] Sadgrove M, Wimberger S, Parkins S and Leonhardt R 2005 Phys. Rev. Lett. 94174103

[13] Bharucha CF, Robinson JC, Moore FL, Sundaram B, Niu Q and Raizen MG 1999 Phys. Rev. E 603881

[14] Casati G, Chiriko BV, Izrailev F and Ford J in Stochastic Behavior in Classical and Quantum Hamiltonian Systems, ed. by G. Casati and J. Ford 1979 (Springer, Berlin); Fishman S, in Quantum Chaos, Proceedings of the International School of Physics "Enrico Fermi" CXIX, eds. G. Casati et al. 1993 (IOS, Amsterdam)

[15] Izrailev FM 1990 Phys. Rep. 196299

[16] Fishman S, Guarneri I and Rebuzzini L 2002 Phys. Rev. Lett. 89 084101; 2003 J. Stat. Phys. 110 911

[17] Wimberger S, Guarneri I and Fishman S 2004 Phys. Rev. Lett. 92084102

[18] Graham R, Schlautmann M and Zoller P 1992 Phys. Rev. A 45 R19

[19] Sadgrove M, Mullins T, Parkins S and Leonhardt R 2005 Phys. Rev. E 71027201

[20] Blümel R, Fishman S and Smilansky U 1986 J. Chem. Phys. 842604

[21] Klappauf BG, Oskay WH, Steck DA and Raizen MG 1998 Physica D 13178

[22] Chirikov BV 1979 Phys. Rep. 52264

[23] Wimberger S, Sadgrove M, Parkins S and Leonhardt R 2005 Phys. Rev. A 71053404

[24] Duffy G, Parkins S, Müller T, Sadgrove M, Leonhardt R and Wilson AC 2004 Phys. Rev. E 70 056206

[25] Wimberger S, Mannella R, Morsch O and Arimondo E 2005 Phys. Rev. Lett. 94130404

Rebuzzini L, Wimberger S and Artuso R 2005 Phys. Rev. E 71036220 